\title{
Distribution Profiles of Membrane Type-1 Matrix Metalloproteinase (MT1-MMP), Matrix Metalloproteinase-2 (MMP-2) and Cyclooxygenase- 2 (COX-2) in Rabbit Atherosclerosis: Comparison with Plaque Instability Analysis
}

\author{
Yuji Kuge, ${ }^{*}, a$ Nozomi Takai, ${ }^{a}$ Seigo Ishino, ${ }^{a}$ Takashi Temma, ${ }^{a}$ Masashi Shiomi,${ }^{b}$ and Hideo SAJI ${ }^{a}$ \\ ${ }^{a}$ Department of Patho-functional Bioanalysis, Graduate School of Pharmaceutical Sciences, Kyoto University; 46-29 \\ Yoshida Shimoadachi-cho, Sakyo-ku, Kyoto 606-8501, Japan: and ${ }^{b}$ Institute for Experimental Animals, Kobe University \\ School of Medicine; 7-5-1 Kusunokicho, Chuo-ku, Kobe 650-0017, Japan. \\ Received March 3, 2007; accepted June 27, 2007; published online July 6, 2007
}

\begin{abstract}
Background: Despite increasing evidence that membrane type 1 matrix metalloproteinase (MT1-MMP), matrix metalloproteinase-2 (MMP-2), and cyclooxygenase-2 (COX-2) are involved in the pathogenesis of atherosclerosis, the possible links among these enzymes remain unclear. Accordingly, we investigated the distribution of MT1-MMP, MMP-2, and COX-2 immunohistologically in the atherosclerotic lesions of hypercholesterolemic (WHHLMI) rabbits. Methods and Results: Distribution of MT1-MMP, MMP-2, and COX-2 was examined by immunohistochemical staining using sixty cross sections of the ascending-arch and thoracic aortas prepared from 4 WHHLMI rabbits. MT1-MMP and MMP-2 staining was prominently observed in the macrophage-rich regions of the atheromatous lesions, and was positively correlated with morphological vulnerability $(r=0.63$ for MT1-MMP; $r=0.60$ for MMP-2; $\boldsymbol{p}<\mathbf{0 . 0 0 0 1 )}$. MT1-MMP staining was positively correlated with MMP-2 staining $(r=0.61, p<0.0001)$. COX-2 staining was also the highest in the macrophage-rich regions of the atheromatous lesions, with relatively high staining levels in other more stable lesions. Conclusions: Co-distribution of MT1MMP, MMP-2, and COX-2 was demonstrated in grade IV atheroma, indicating a possible link among these enzymes in the destabilization of atherosclerotic plaques. The relatively high COX-2 distribution in other more stable lesions may indicate its additional roles in the stabilization of atherosclerotic lesions. The present findings in hypercholesterolemic rabbits should help advance our understanding of the pathophysiology of atherosclerosis and provide useful information for the development of new therapeutic and diagnostic (imaging) agents that target MMPs and COX-2 in atherosclerosis.
\end{abstract}

Key words atherosclerosis; matrix metalloproteinase; cyclooxygenase; rabbit; plaque

Our current understanding of the pathophysiology of atherosclerosis suggests the involvement of complex mechanisms that go beyond mere lipid storage disorders. ${ }^{1,2)}$ It is of great importance to investigate causative factors in the destabilization of atherosclerotic plaques, which should help develop new therapeutic and diagnostic (imaging) agents of atherosclerosis, leading to the establishment of novel therapeutic strategies for preventing acute coronary syndromes and stroke.

To date, several factors, including enhanced inflammatory responses and expression of matrix metalloproteinases (MMPs), have been suggested to play important roles in the destabilization of atherosclerotic plaques. ${ }^{1-4)}$ Plaques prone to rupture are morphologically characterized by a thin fibrous cap overlying a large lipid core. MMPs have been shown to degrade extracellular matrix (ECM) that constitutes the fibrous cap of the plaques, resulting in the destabilization of atherosclerotic plaques. ${ }^{3-5)}$ Increased expression of MMP-2 and MMP-9 has been demonstrated within human atherosclerotic lesions and critically implicated in plaque rupture. ${ }^{5-7)}$ MMP-2 and MMP-9 are known to cleave native type IV, V, VII, and X collagens and elastin, as well as the products of collagens types I, II, and III after proteolysis by collagenases, such as MMP-1 and MMP-13, and are considered to be involved in plaque instability. ${ }^{3}$ The MMPs can be divided into two groups: soluble MMPs and membranebound MMPs. Most soluble MMPs, including MMP-2 and MMP-9, are released from cells as zymogens and require extracellular posttranslational cleavage to gain biological activ- ity. ${ }^{3,8)}$ A membrane-bound MMP, membrane type-1 MMP (MT1-MMP or MMP-14), has been demonstrated to mediate the activation of pro-MMP-2 to active MMP-2 on the cell surface. ${ }^{8,9)}$ The expression of MT1-MMP has also been found within human atherosclerotic plaques. ${ }^{10,11)}$ Thus, MT1MMP, an activator of pro-MMP-2 to active MMP-2, has been speculated to be an important determinant of the destabilization of atherosclerotic plaques.

Production of MMP-2 and MMP-9 by monocytes/ macrophages occurs through a prostaglandin (PG) $\mathrm{E}_{2} /$ cAMP-dependent pathway. ${ }^{1,12,13)}$ Recently, Shankavaram et al. demonstrated that induction of monocyte MT1-MMP is also regulated through the $\mathrm{PG} \mathrm{E}_{2} / \mathrm{cAMP}$ pathway. ${ }^{14)}$ These findings indicate the involvement of cyclooxygenases (COXs), rate-limiting enzymes in the conversion of arachidonic acid into PGs and thromboxanes, in the regulation of MMP biosynthesis. ${ }^{1,15}$ To date, at least 2 distinct isoforms of the COXs - a constitutive form (COX-1) and an inducible isoform (COX-2) - and several of their variants have been discovered. ${ }^{16)}$ COX-1 is constitutively expressed in most tissues and is responsible for maintaining homeostasis, whereas COX-2 is induced in response to inflammatory stimuli. ${ }^{17)}$ Overexpression of COX-2 has been shown within human atherosclerotic plaques, localized predominantly in macrophages/foam cells, and to a lesser extent in medial smooth muscle cells and endothelial cells. ${ }^{18,19)}$ Extensive studies by Cipollone et al. have provided evidence that COX-2 expression is associated with acute ischemic syndromes, possibly through MMP-2/MMP-9 induced plaque 
rupture. ${ }^{3,12,20)}$ Hong et al. reported the coexpression of COX2 and MT1-MMP in the atherosclerotic intima, plaque itself, and vascular smooth muscle cells in patients with atherosclerotic aortic aneurysm and dissection. ${ }^{21)}$

Taken together, the signaling cascade, namely, activation of pro-MMP-2 to active MMP-2 by MT1-MMP through the $P G E_{2}$ /cAMP pathway, may play pivotal roles in the destabilization of atherosclerotic plaques. However, the roles of the possible links among MT1-MMP, MMP-2, and COX-2 in the pathophysiology of atherosclerosis have not been fully investigated. To the best of our knowledge, there has been no report that directly compared the distribution profiles of MT1MMP, MMP-2, and COX-2 in atherosclerotic lesions in vivo.

Watanabe heritable hyperlipidemic (WHHL) rabbits and a current strain of the rabbits, myocardial infarction-prone Watanabe heritable hyperlipidemic (WHHLMI) rabbits, have been widely used as animal models of spontaneous atherosclerosis because the pathological characteristics have been reported to be relevant to human atherosclerosis. ${ }^{22,23)}$ The use of WHHLMI rabbits could be effective for investigating causative factors in the destabilization of atherosclerotic plaques, and should provide a useful means for investigating new therapeutic and diagnostic (imaging) agents of atherosclerosis. Thus, in the present study, we investigated the distribution of MT1-MMP, MMP-2, and COX-2 immunohistologically in the atherosclerotic lesions of WHHLMI rabbits, in comparison with atherosclerotic plaque instability analysis.

\section{MATERIALS AND METHODS}

Animals Four female WHHLMI rabbits (12.6 \pm 0.8 months old: $3.7 \pm 0.3 \mathrm{~kg}$ body weight) bred at Kobe University were used in the present study. Four Japanese White rabbits $(3.1 \pm 0.1$ months old: $2.7 \pm 0.1 \mathrm{~kg}$ body weight) purchased from Biotec. Inc. (Saga, Japan) were also used as controls. The rabbits were fed standard rabbit chow (type CR-3; Clea Japan Inc., Tokyo, Japan: $120 \mathrm{~g} / \mathrm{d}$ ) and were given water ad libitum. All experimental procedures were approved by the Kyoto University Animal Care Committee.

Preparation of Histological Sections The rabbits were sacrificed with an overdose of sodium pentobarbital. The ascending-arch and thoracic aorta were cut into 6 and 9 segments, respectively. Each segment was immediately fixed in a solution containing $\mathrm{L}-(+)$-lysine hydrochloride $(75 \mathrm{mmol} / \mathrm{l})$ and $4 \%$ paraformaldehyde in phosphate buffer $(37.5 \mathrm{mmol} / \mathrm{l}$; $\mathrm{pH} 7.4$ ), and embedded in paraffin. Consecutive $5-\mu \mathrm{m}$-thick slices were prepared at the center of each segment.

Histological Analysis Serial sections were subjected to immunohistochemical staining for MT1-MMP, MMP-2, COX-2 and cell type marker antigens, as well as Azan-Mallory and hematoxylin-eosin (HE) staining. Immunohistochemical staining was performed according to standard immunostaining procedures with slight modifications. ${ }^{21,24-26)}$ MT1-MMP and MMP-2 were immunostained with a purified mouse monoclonal antibody to an oligopeptide (residue 319 to 333 , numbered from signal peptide) on human MT1-MMP ( 1 : 50 dilution; 113-5B7, mouse IgG, Daiichi Fine Chemical Co., Ltd., Toyama, Japan) and with that to an oligopeptide (residue 468 to 483 , numbered from propeptide) on human MMP-2 (1:20 dilution; 42-5D11, mouse IgG, Daiichi Fine
Chemical Co., Ltd.), respectively. These antibodies specifically recognize both pro- and active forms of the enzymes. Immunostainings for a rabbit macrophage-specific antigen (1:50 dilution; RAM-11, mouse IgG) and smooth muscle actin (1:50 dilution; 1A4, mouse IgG) were also performed using monoclonal antibodies obtained from Dako Corp., Santa Barbara, CA, U.S.A. The bound antibodies were visualized by using a DAKO Envision + kit (Dako) and 3,3'-diaminobenzidine tetrahydrochloride (DAB) (Dako). Counterstaining with hematoxylin was performed. Immunostaining with subclass-matched irrelevant IgG served as negative controls.

For COX-2 immunostaining, deparaffinized sections were heated with a microwave oven for antigen retrieval. Thereafter, the specimens were incubated with a goat polyclonal antibody (1:200 dilution; sc-1745, Santa Cruz Biotechnology, Inc., CA, U.S.A.) that raised against a peptide sequence at the $\mathrm{C}$-terminus of human COX-2. The bound antibody was visualized using a Dako LSAB + kit (Dako) with hematoxylin counterstaining.

Classification of Atherosclerotic Lesions The atherosclerotic lesions in WHHLMI rabbits were divided into 4 categories using a classification scheme based on the recommendations of the American Heart Association (AHA) ${ }^{27,28)}$ by Azan-Mallory and HE staining, as previously described (13): (1) neointimal lesion (Type I-III), (2) atheromatous lesion (Type IV), (3) fibroatheromatous lesion (Type Va, Vb), (4) collagen-rich lesion (Type Vc), as shown in Fig. 2. Neointimal lesions were defined as having adaptive thickening of the intima consisting mainly of smooth muscle cells (SMCs) and few macrophages. Atheromatous lesions contained thin fibrous connective tissue and a dense accumulation of extracellular lipid and foam cells, and were considered to be vulnerable-like lesions in human atherosclerotic plaques. Fibroatheromatous lesions were composed of several lipid cores and separated by thick layers of fibromuscular connective tissue, which was relatively stable to rupture. ${ }^{29)}$ Collagen-rich lesions consisted of a predominantly collagenous component and contained smooth muscle cells. The distinction between the fibroatheromatous and collagenrich lesions was made mainly based on the inclusion and exclusion of lipid cores. Extracellular vacuoles and lacunae on the Azan-Mallory and HE stained specimens were considered to be lipid cores.

In the ascending-arch and thoracic aortas of WHHLMI rabbits, a total of 191 histopathological features which correspond to the classification criteria were observed (neointimal, $n=14$; atheromatous, $n=44$; fibroatheromatous, $n=56$, and collagen-rich, $n=77$ ). There were no lesions showing plaque rupture or thrombi (type VI) in the present study. Thus, the 191 regions were divided into the 4 lesion-categories and semi-quantitatively evaluated in subsequent analyses.

Semi-quantitative Analyses Areas $\left(\mu \mathrm{m}^{2}\right)$ occupied by each lesion component were evaluated with a VHX Digital Microscope (Keyence Corp., Osaka, Japan). The vulnerability index, an index of the morphological destabilized characteristics of atherosclerotic lesions in WHHLMI rabbits, was calculated for each atherosclerotic region as previously described. ${ }^{24,30)}$ The vulnerability index was defined as the ratio of the lipid component area (macrophages+extracellular lipid deposits)/fibromuscular component area (smooth mus- 
cle cells + collagen fibers). Collagen-rich fibers and extracellular lipid deposits (extracellular vacuoles and lacunae) were assessed with Azan-Mallory staining. The macrophage and smooth muscle cell areas were determined with immunohistochemical staining (RAM11 and 1A4). MT1-MMP, MMP-2, and COX-2 staining were assessed as percentages of positively stained areas ( $\%$ positive).

Statistical Analyses Data are presented as the meant S.D. Comparisons among lesion types were performed using the Kruskal-Wallis test with post hoc analysis by the Scheffe test. Correlation coefficients were assessed with Spearman rank correlation coefficients. Comparisons of correlation coefficients were performed using Fisher's Z-transformation. Statistical significance was defined as $p<0.05$.

\section{RESULTS}

MT1-MMP, MMP-2 and COX-2 Distribution in Atherosclerotic Lesions Figure 1 shows representative photomicrographs of aortic tissues of the control rabbits. No obvious atherosclerotic changes were observed in the control rabbits.

In the WHHLMI rabbits, various atherosclerotic changes were observed with different staining levels of MT1-MMP, MMP-2, and COX-2. Figure 2 shows typical images of the 4 categories of lesion types with Azan-Mallory, HE and immunohistochemical staining. MT1-MMP staining was prominent in the atheromatous lesions, which were also the regions where macrophages were accumulated (Figs. 2J, R). Weak staining of MT1-MMP was observed in the lipid core regions of fibroatheromatous lesions and superficial regions of collagen-rich lesions, with no obvious staining in the neointimal lesions or medial regions (Figs. 2Q-T). The staining profile of MMP-2 was similar to that of MT1-MMP, except for relatively high staining of MMP-2 in the medial regions (Figs. $2 \mathrm{U}-\mathrm{X})$. COX-2 staining was widely observed in the atherosclerotic lesions of the WHHLMI rabbits. Strong COX-2 staining was detected in the macrophage-rich regions of the atheromatous and fibroatheromatous lesions. Weak to moderate COX-2 staining was detected also in the superficial regions of the neointimal and collagen-rich lesions (Figs. $2 \mathrm{Y}-\mathrm{b})$.

Highly magnified photomicrographs of immunostaining for the lipid core and medial regions of atheromatous lesions are shown in Fig. 3. MT1-MMP, MMP-2, and COX-2 staining was prominently observed in the lipid core regions colocalizing with macrophage staining (Figs. 3A-D). Relatively strong staining of MMP-2 was observed in the medial regions where smooth muscle actin was positively stained (Figs. 3F, H).

Semi-quantitative Analyses of MT1-MMP, MMP-2 and COX-2 Distribution in Relation to Plaque Vulnerability Figure 4A shows the vulnerability index calculated for each lesion category classified as described in Materials and Methods. This index was the highest in the atheromatous lesions ( $p<0.0001 v s$. other lesions), followed in decreasing order by the fibroatheromatous lesions $(p<0.005$ vs. neointimal lesions; $p<0.0001$ vs. collagen-rich lesions), collagenrich lesions, and neointimal lesions. MT1-MMP staining (\% positive) was the highest in the atheromatous lesions, where it was 10.9-, 3.5-, and 6.3-fold higher than that in the neointimal, fibroatheromatous, and collagen-rich lesions, respec- tively ( $p<0.0001 v s$. other lesions, Fig. 4B). MMP-2 staining ( $\%$ positive) was also the highest in the atheromatous lesions among the four lesion types: 4.8-, 2.5-, and 4.2-fold higher than that in the neointimal, fibroatheromatous, and collagenrich lesions, respectively $(p<0.0001 v s$. other lesions, Fig. $4 \mathrm{C})$. The highest staining of COX-2 ( $\%$ positive) was also found in the atheromatous lesions, while comparable staining levels were observed in the neointimal and fibroatheromatous lesions (Fig. 4D). The \% positive area of COX-2 in the atheromatous lesions was 1.2-, 1.3-, and 1.7-fold that in the neointimal, fibroatheromatous, and collagen-rich lesions, respectively (N.S., vs. neointimal and fibroatheromatous lesions; $p<0.0001$ vs. collagen-rich lesions).

Analyses of Correlations Among MT1-MMP, MMP-2 and COX-2 Staining and Histological Vulnerability Analysis of the correlation of the vulnerability index with MT1-MMP, MMP-2 and COX-2 staining (\% positive) is shown in Figs. 5A-C. MT1-MMP staining was positively correlated with the vulnerability index $(r=0.63, p<0.0001$, Fig. 5A). Similarly, MMP-2 and COX-2 staining was positively correlated with the vulnerability index $(r=0.60, p<$ 0.0001 for MMP-2; $r=0.36, p<0.0001$ for COX-2, Figs. $5 \mathrm{~B}, \mathrm{C})$. The correlation of the vulnerability index with MT1MMP and MMP-2 staining was significantly stronger than that with COX-2 staining ( $p<0.05$, for each).

Figures 5D-F show the regression analyses among MT1MMP, MMP-2 and COX-2 staining (\% positive). The regression analyses demonstrated positive correlations between MT1-MMP and MMP-2 staining $(r=0.61, p<0.0001$, Fig. 5D), between MT1-MMP and COX-2 staining ( $r=0.33$, $p<0.0001$, Fig. 5E), and between MMP-2 and COX-2 staining $(r=0.46, p<0.0001$, Fig. 5F). The correlation between MT1-MMP and MMP-2 staining was significantly stronger than those between MT1-MMP and COX-2 and between MMP-2 and COX-2 ( $p<0.05$, for each).

\section{DISCUSSION}

In the present study, we first demonstrated the co-distribution of MT1-MMP, MMP-2, and COX-2 in atherosclerotic lesions. The preferential distribution of these enzymes in the atheromatous lesions (grade IV atheroma) provides in vivo evidence for the possible interaction among these enzymes in the destabilization of atherosclerotic plaques. Relatively high COX-2 distribution was also found in other more stable lesions, indicating additional roles of $\mathrm{COX}-2$ in the stabilization of atherosclerotic lesions.

Colocalization of MT1-MMP, MMP-2, and COX-2 in Grade IV Atheroma Recent evidence about the pathophysiology of atherosclerosis suggests that a signaling cascade, namely, activation of pro-MMP-2 to active MMP-2 by MT1-MMP through the PG $E_{2}$ /cAMP pathway, may play pivotal roles in the destabilization of atherosclerotic plaques. However, there has been no direct evidence regarding the interactions of MT1-MMP, MMP-2, and COX-2 in atherosclerotic lesions. In the present study, we immunohistologically demonstrated the colocalization of MT1-MMP, MMP-2, and COX-2 in the atheromatous lesions (grade IV atheroma) with the highest vulnerability index (Figs. 2 to 4). In addition, these enzymes were prominently localized in the macrophage-rich lipid core region of the grade IV atheroma 


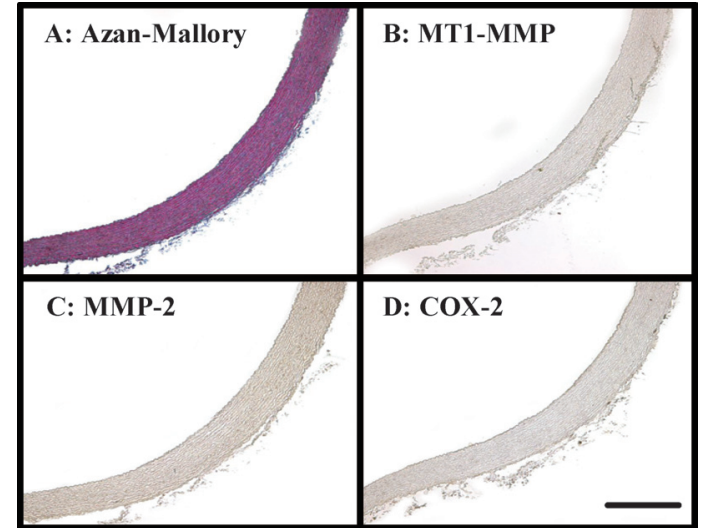

Fig. 1. Photomicrographs of Aortic Tissues of the Control Rabbit

Azan-Mallory staining (A) and immunohistochemical staining for MT1-MMP (B), MMP-2 (C), and COX-2 (D) are shown. Bar $=500 \mu \mathrm{m}$.

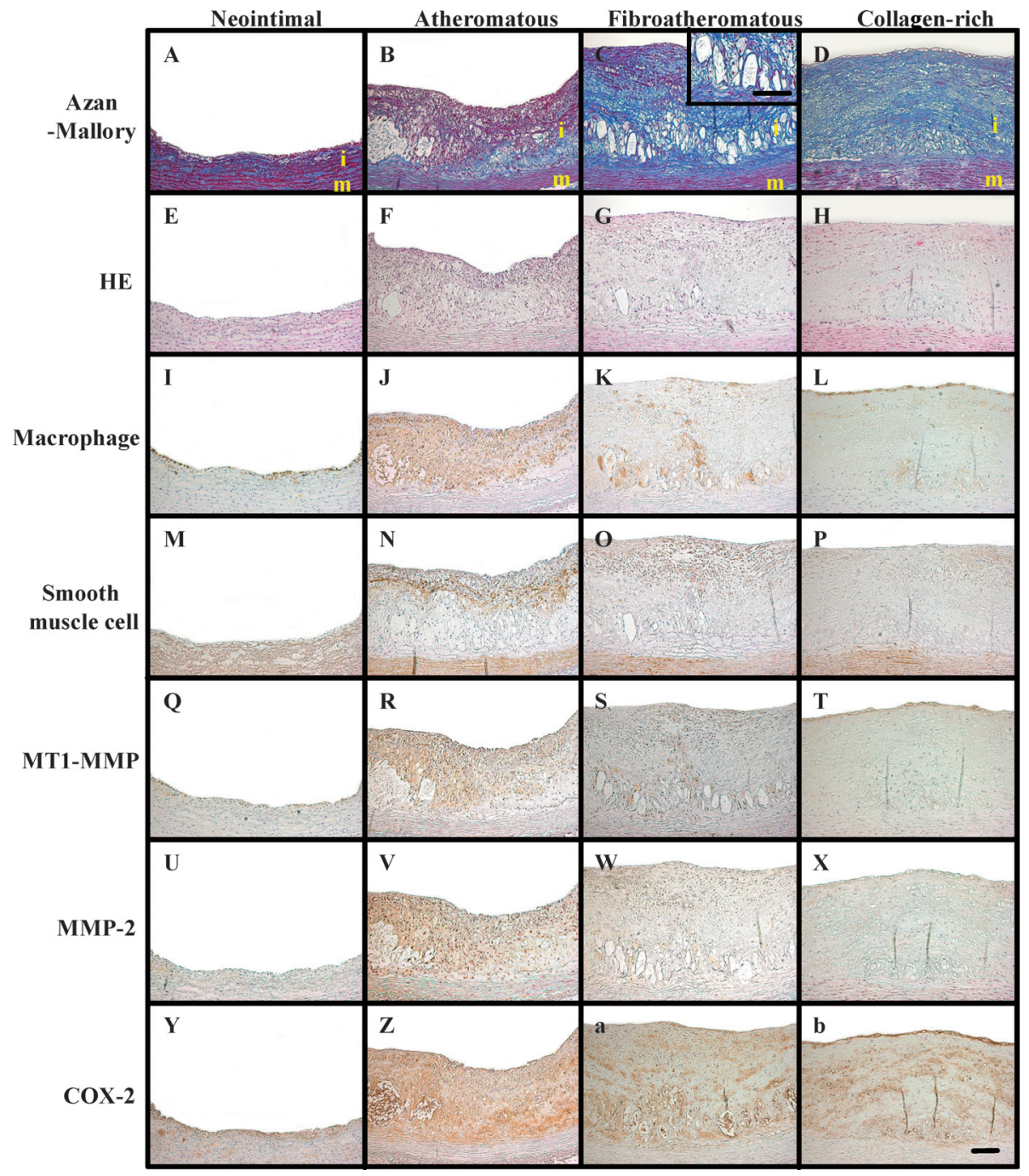

MT1-MMP

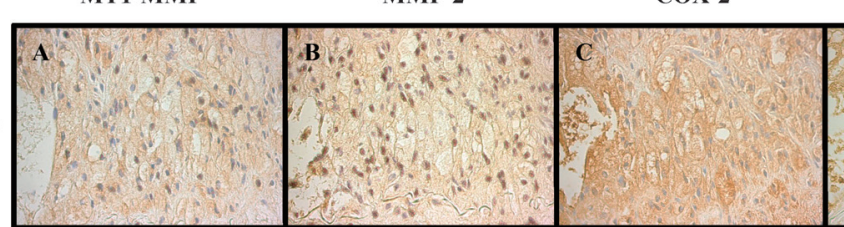

Lipid core

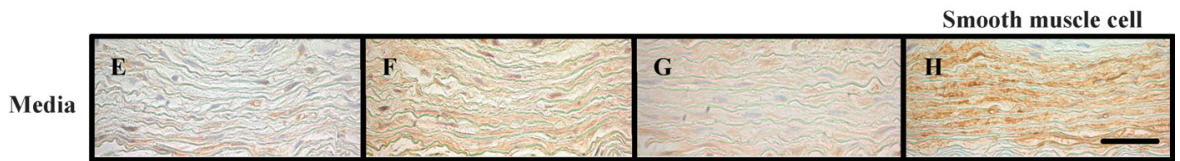

Fig. 2. Typical Photomicrographs of Histological Features of Atherosclerotic Lesions in WHHLMI Rabbits

Atherosclerotic lesions were microscopically divided into 4 categories as described in Materials and Methods: neointimal (left column), atheromatous (middle-left column), fibroatheromatous (middleright column) and collagen-rich (right column) lesions. Azan-Mallory staining (A to D), HE staining (E to $\mathrm{H}$ ), and immunostaining for macrophages (I to L), smooth muscle cells (M to P), MT1-MMP (Q to $\mathrm{T}$ ), MMP-2 (U to $\mathrm{X}$ ), and COX-2 ( $\mathrm{Y}$ to b) are shown. A high-magnification photomicrograph of Azan-Mallory staining in the lipid core region is shown in an inset (C). m, media; i, intima; $\mathrm{Bar}=100 \mu \mathrm{m}$.

Fig. 3. High-Magnification Photomicrographs of Immunohistochemical Staining for MT1-MMP (A, E), MMP-2 (B, F), COX-2 (C, G), Macrophages (D), and Smooth Muscle Cells (H) in the Lipid Core (A to D) and Medial (E to H) Regions of the Atheromatous Lesions Shown in Fig. 2

$\mathrm{Bar}=50 \mu \mathrm{m}$. 


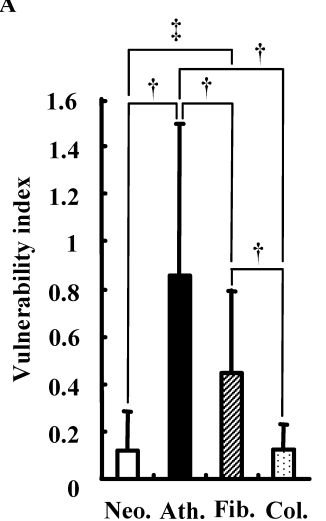

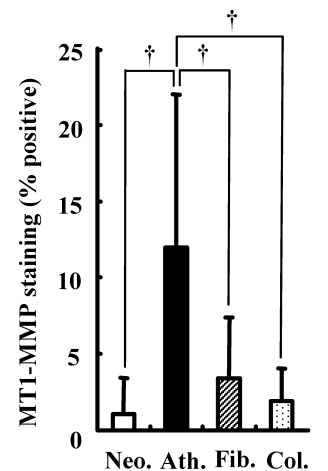

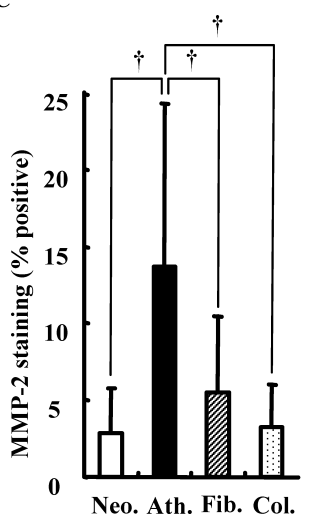

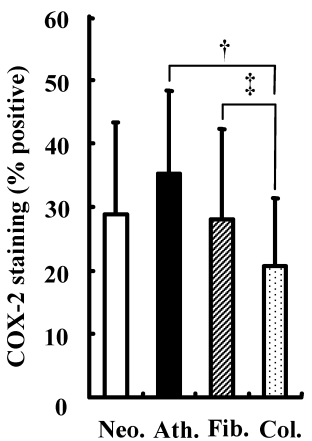

Fig. 4. Vulnerability Index (A) and Percentages of Positively Stained Areas (\% Positive) for MT1-MMP (B), MMP-2 (C), and COX-2 (D) in Atherosclerotic Lesions

Neo., Ath., Fib. and Col. indicate neointimal, atheromatous, fibroatheromatous and collagen-rich lesions, respectively. Data are presented as the mean \pm S.D. Comparisons among lesion types were performed using the Kruskal-Wallis test with post hoc analysis by the Scheffe test. $\dagger p<0.0001, \ddagger p<0.05$.
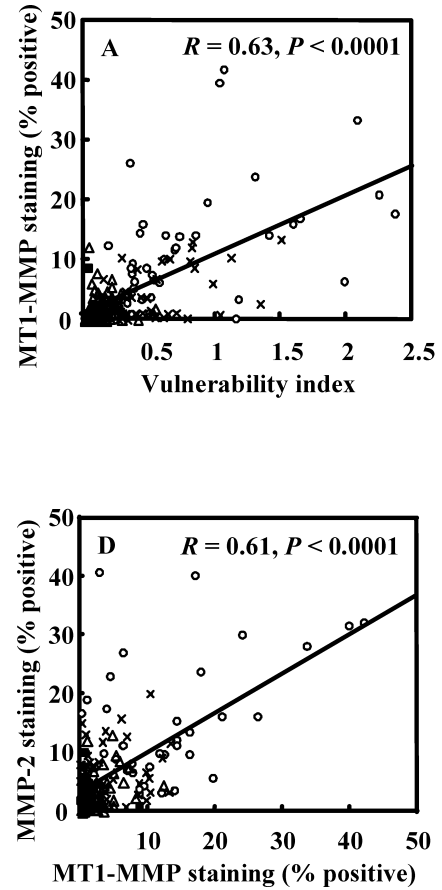
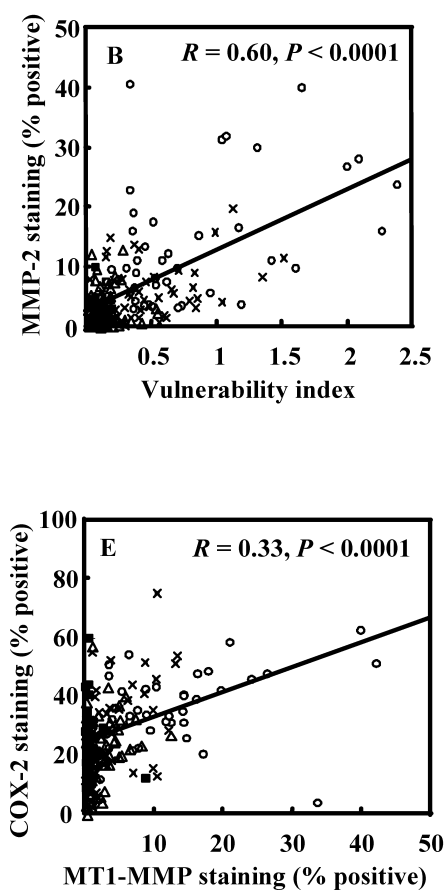
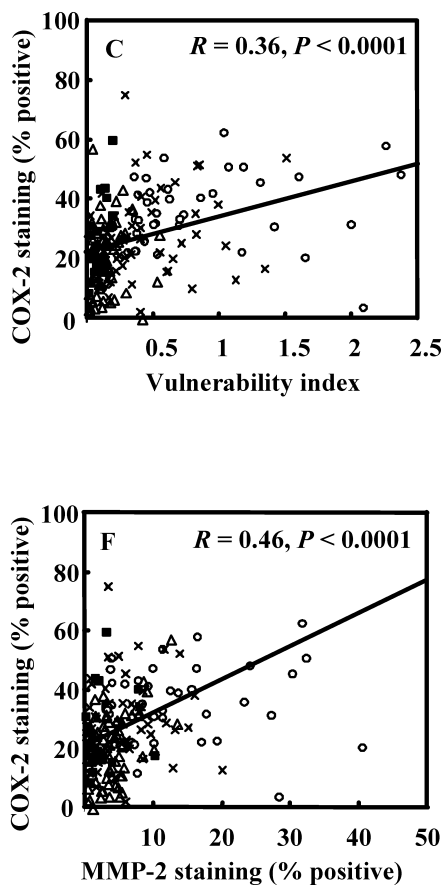

Fig. 5. Analyses of Correlations among MT1-MMP, MMP-2 and COX-2 Staining and Histological Vulnerability

口; neointimal, $\bigcirc$; atheromatous, $\times$; fibroatheromatous, $\triangle$; collagen-rich lesions. A-C: Correlations of vulnerability index with MT1-MMP (A), MMP-2 (B), and COX-2 (C) staining (\% positive), D-F: Correlations among MT1-MMP, MMP-2 and COX-2 staining (\% positive). Correlation coefficients were assessed with Spearman rank correlation coefficients. Comparisons of correlation coefficients were performed using Fisher's Z-transformation.

(Figs. 2, 3). These findings support the possibility of interactions among MT1-MMP, MMP-2, and COX-2 and their contribution to plaque progression and rupture.

Distribution of MT1-MMP/MMP-2 In the present study, we investigated MT1-MMP distribution in a rabbit model of atherosclerosis. To the best of our knowledge, this is the first report demonstrating MT1-MMP distribution in atherosclerotic lesions of an animal model. The distribution profiles of MT1-MMP and MMP-2 in our rabbits are mostly consistent with the previous clinical studies that demonstrated a notable colocalization of MT1-MMP and MMP2 with macrophages in lipid-rich human atherosclerotic plaques. ${ }^{10,11)}$ In addition, the current results demonstrated that MT1-MMP distribution was positively correlated with vulnerability index and MMP-2 distribution (Fig. 5). Ac- cordingly, our findings further support the contribution of MT1-MMP/MMP-2 to the destabilization of atherosclerotic plaques. ${ }^{10,11)}$

It should be noted, however, that dual roles of MMPs have been well documented. ${ }^{31)}$ MMP activities not only contribute to weakening of the plaque cap via cleavage of the ECM but also determine lesion stability through the cleavage of nonmatrix substrates, including cytokines, growth factors, and their receptors. ${ }^{4,8,11)}$ Recently, Kuzuya et al. examined the effects of MMP-2 deficiency on atherosclerotic lesion formation in apolipoprotein E-deficient $(\mathrm{ApoE}-/-)$ mice. ${ }^{32)}$ Based on their results, they suggested that MMP-2 may induce plaque stability by promoting the accumulation of SMCs in the fibrous cap, while they showed different effects of MMP2 on the macrophage accumulation in the atherosclerotic le- 
sions of different regions of the artery. Further studies will be required to determine the full spectrum of antiatherogenic or proatherogenic activities of MMPs expressed in atherosclerotic lesions.

In our rabbits, no significant staining of MT1-MMP was found in the medial regions (SMCs) with slight to moderate staining of MMP-2. Contrary to our results, previous clinical studies showed a notable distribution of MT1-MMP and MMP-2 in the media (SMCs) underlying fibrous and lipidrich regions, suggesting a contribution of MT1-MMP and MMP-2 to SMC-mediated vascular remodeling. ${ }^{10,11)}$ In vitro experiments have also shown that MT1-MMP expression increases not only in macrophages but also in SMCs after proinflammatory stimulation. ${ }^{10,11,33)}$ Although the reason for the discrepancy remains unclear, differences in species, age, and stage of atherosclerosis may partly explain the discrepancy. Distribution profiles of MMPs may change according to age and stage of atherosclerosis. ${ }^{4,31)}$ On the other hand, it is reported that MT1-MMP provided by macrophages may play a significant role in the activation of MMP-2 produced by other cells, such as SMCs. ${ }^{114)}$ MMP-2 is constitutively expressed in medial SMCs. ${ }^{31}$ These facts may be another explanation for the uncoupling of MT1-MMP and MMP-2 distribution in the medial regions (SMCs).

Distribution of COX-2 in Relation to MT1-MMP Distribution Although there is increasing evidence that COX2 plays an important role in the pathophysiology of atherosclerotic plaques, the relationship between MT1-MMP and COX-2 has been poorly understood. In particular, there has been no report investigating in vivo co-distribution of these enzymes in atherosclerotic lesions, except for one clinical study reported by Hong et $a .^{21)}$ Our present study demonstrated relatively strong staining of MT1-MMP and COX-2 in the macrophage-rich regions of the atheromatous and fibroatheromatous lesions in a rabbit model, which is concordant with the clinical results reported by Hong et al. These results are further supported by the previous in vitro findings of the enhanced expression of MT1-MMP in human macrophages after proinflammatory stimulation, such as ox-LDL or TNF- $\alpha .^{11,33)}$ Shankavaram et al. also demonstrated that induction of monocyte MT1-MMP is regulated through the $\mathrm{PG} \mathrm{E}_{2} / \mathrm{cAMP}$ pathway, indicating the important role of COX-2 in the regulation of MT1-MMP. ${ }^{14)}$ Taken together, these facts imply that MT1-MMP production through the COX-2 dependent pathway may also promote plaque instability, as does the production of other MMPs, including MMP-2 and MMP-9. ${ }^{12,20)}$

In our WHHLMI rabbits, relatively strong COX-2 staining was found also in more stable atherosclerotic lesions, such as neointimal and collagen-rich lesions (Figs. 2, 3), indicating that the distribution of COX-2 and MT1-MMP/MMP-2 was uncoupled in these lesions. In addition, the correlation of COX-2 staining with the vulnerability index was significantly weaker compared with those of MT1-MMP and MMP-2 (Fig. 5). This COX-2 distribution profile is in accordance with previous studies where COX-2 distribution was found in early to advanced atherosclerotic lesions in ApoE-/- mice and humans. ${ }^{18,34)}$ The contribution of COX-2 to early atherosclerotic lesion formation has been suggested. ${ }^{34)}$ On the other hand, uncoupling between MT1-MMP and COX-2 distribution has not been reported, and the reason for the uncoupling observed here remains unclear. However, it should be noted that COX-2 is only an intermediate enzyme in the metabolic pathway of arachidonic acid, and that the COX-2 bioproduct $\mathrm{PGH}_{2}$ is further metabolized by other isomerases to various prostanoids. The PG isomerase profile may influence the proinflammatory or anti-inflammatory role of COX-2 and may regulate MMP production in atherosclerotic plaques. ${ }^{15,20)}$ Our findings, namely relatively strong $\mathrm{COX}-2$ staining with weak staining of MT1-MMP in more stable atherosclerotic lesions, appear to support the dual-phase functions of COX-2 reported by Cipollone et al. ${ }^{12,15,20)}$

Methodological Considerations In the present study, WHHLMI rabbits were used to determine the distribution profiles of MT1-MMP, MMP-2 and COX-2 in comparison with plaque instability analysis, because they have several advantages as an animal model for studying atherosclerosis: (1) lipoprotein profile similar to that of humans, (2) susceptibility to the development of atherosclerosis, (3) lesion characteristics (from early to advanced stage) similar to those in humans. ${ }^{22,23,35,36)}$ One drawback in our rabbits is that they failed to show any lesion ruptures or thrombosis, although rupture-prone unstable plaques characterized in humans as those consisting of thin fibromuscular caps and large lipid cores with numerous macrophages were observed in the aortic lesions of our rabbits. The atherosclerotic lesions found in the aortas of our rabbits may not exactly follow the process leading to plaque rupture, which may partly explain the discrepancy between the present results and the previous clinical studies. On the other hand, it is reported that plaque rupture was detected in the coronary lesions of WHHLMI rabbits that died by myocardial infarction, although the frequency was rather low. ${ }^{35)}$ Detail studies in the coronary lesions of WHHLMI rabbits may help clarify the discrepancy between the present results and the previous clinical studies, and provide further important information on the pathophysiology leading to myocardial infarction, as the WHHLI rabbits are the rabbit model of spontaneous myocardial infarction.

The distribution of MT1-MMP, MMP-2, and COX-2 was immunohistologically determined in the atherosclerotic lesions. The immunohistological staining is a useful and widely accepted method for the evaluation of regional distribution of proteins, including enzymes, receptors, and transporters, in human and animal tissues. ${ }^{6,10-12,18,21)}$ The immunohistological staining, however, can not provide information on the synthesis or degradation of the antigen proteins. In this regard, it is of importance to determine the expression levels of corresponding mRNA. Further elucidation, combined with in situ hybridization, is strongly required to determine the dynamic processes of the expression, interaction, and degradation of MT1-MMP, MMP-2, and COX-2. Differential evaluation of pro-MMPs and active-MMPs should also provide useful information regarding the interactions among the enzymes.

\section{CONCLUSION}

The present study demonstrated the co-distribution of MT1-MMP, MMP-2, and COX-2 in grade IV atheroma, using hypercholesterolemic rabbits whose pathological characteristics are relevant to human atherosclerosis. These find- 
ings support our hypothesis that the activation of pro-MMP-2 to active MMP-2 by MT1-MMP through the PG $\mathrm{E}_{2} / \mathrm{cAMP}$ pathway may play pivotal roles in the destabilization of atherosclerotic plaques. In addition, relatively strong COX-2 staining was observed in other more stable lesions, indicating uncoupling between COX-2 and MT1-MMP/MMP-2 distribution. COX-2 may be associated with both the destabilization and stabilization of atherosclerotic lesions via its proand anti-inflammatory activities. The present findings should help advance our understanding of the pathophysiology of atherosclerosis and provide useful information for the development of new therapeutic and diagnostic (imaging) agents that target MMPs and COX-2 in atherosclerosis.

Acknowledgements This work was partly supported by a Grant-in-Aid for General Scientific Research from the Ministry of Education, Culture, Sports, Science and Technology of Japan, by a Grant-in-Aid for General Scientific Research from the Japan Society for the Promotion of Science, by a research grant from New Energy and Industrial Technology Development Organization (NEDO), and by the 21 st Century COE Program 'Knowledge Information Infrastructure for Genome Science'.

\section{REFERENCES}

1) Cipollone F., Fazia M., Mezzetti A., J. Thromb. Haemost., 3, 1962 1975 (2005).

2) Stoll G., Bendszus M., Stroke, 37, 1923-1932 (2006).

3) Jones C. B., Sane D. C., Herrington D. M., Cardiovasc. Res., 59, $812-823$ (2003).

4) Galis Z. S., Khatri J. J., Circ. Res., 90, 251-262 (2002).

5) Galis Z. S., Sukhova G. K., Lark M. W., Libby P., J. Clin. Invest., 94, 2493-2503 (1994).

6) Brown D. L., Hibbs M. S., Kearney M., Loushin C., Isner J. M., Circulation, 91, 2125-2131 (1995).

7) Li Z., Li L., Zielke H. R., Cheng L., Xiao R., Crow M. T., StetlerStevenson W. G., Froehlich J., Lakatta E. G., Am. J. Pathol., 148, $121-128$ (1996).

8) Visse R., Nagase H., Circ. Res., 92, 827-839 (2003).

9) Sato H., Takino T., Okada Y., Cao J., Shinagawa A., Yamamoto E., Seiki M., Nature (London), 370, 61-65 (1994).

10) Rajavashisth T. B., Xu X. P., Jovinge S., Meisel S., Xu X. O., Chai N. N., Fishbein M. C., Kaul S., Cercek B., Sharifi B., Shah P. K., Circulation, 99, 3103-3109 (1999).

11) Stawowy P., Meyborg H., Stibenz D., Stawowy N. B. P., Roser M., Thanabalasingam U., Veinot J. P., Chretien M., Seidah N. G., Fleck E., Graf K., Circulation, 111, 2820-2827 (2005).

12) Cipollone F., Prontera C., Pini B., Marini M., Fazia M., De Cesare D., Iezzi A., Ucchino S., Boccoli G., Saba V., Chiarelli F., Cuccurullo F., Mezzetti A., Circulation, 104, 921-927 (2001).

13) Corcoran M. L., Stetler-Stevenson W. G., DeWitt D. L., Wahl L. M.,
Arch. Biochem. Biophys., 310, 481-488 (1994).

14) Shankavaram U. T., Lai W. C., Netzel-Arnett S., Mangan P. R., Ardans J. A., Caterina N., Stetler-Stevenson W. G., Birkedal-Hansen H., Wahl L. M., J. Biol. Chem., 276, 19027-19032 (2001).

15) Cipollone F., Fazia M., Iezzi A., Ciabattoni G., Pini B., Cuccurullo C., Ucchino S., Spigonardo F., De Luca M., Prontera C., Chiarelli F., Cuccurullo F., Mezzetti A., Arterioscler. Thromb. Vasc. Biol., 24, 12591265 (2004).

16) Davies N. M., Good R. L., Roupe K. A., Yanez J. A., J. Pharm. Pharm. Sci., 7, 217-226 (2004).

17) Matsumoto H., Naraba H., Murakami M., Kudo I., Yamaki K., Ueno A., Oh-ishi S., Biochem. Biophys. Res. Commun., 230, 110-114 (1997).

18) Baker C. S., Hall R. J., Evans T. J., Pomerance A., Maclouf J., Creminon C., Yacoub M. H., Polak J. M., Arterioscler. Thromb. Vasc. Biol., 19, 646-655 (1999).

19) Schonbeck U., Sukhova G. K., Graber P., Coulter S., Libby P., Am. J. Pathol., 155, 1281-1291 (1999).

20) Cipollone F., Lupus, 14, 756-759 (2005).

21) Hong B. K., Kwon H. M., Lee B. K., Kim D., Kim I. J., Kang S. M., Jang Y., Cho S. H., Kim H. K., Jang B. C., Cho S. Y., Kim H. S., Kim M. S., Kwon H. C., Lee N., Yonsei Med. J., 41, 82-88 (2000).

22) Shiomi M., Ito T., Tsukada T., Yata T., Ueda M., Arterioscler. Thromb., 14, 931-937 (1994).

23) Shiomi M., Ito T., Yamada S., Kawashima S., Fan J., Arterioscler Thromb. Vasc. Biol., 23, 1239-1244 (2003).

24) Ishino S., Kuge Y., Takai N., Tamaki N., Strauss H. W., Blankenberg F. G., Shiomi M., Saji H., Eur. J. Nucl. Med. Mol. Imaging., 34, 889899 (2007)

25) Kinoh H., Sato H., Tsunezuka Y., Takino T., Kawashima A., Okada Y., Seiki M., J. Cell Sci., 109, 953-959 (1996).

26) Tatsuguchi A., Fukuda Y., Ishizaki M., Yamanaka N., Digestion, 60, 246-254 (1999).

27) Stary H. C., Chandler A. B., Glagov S., Guyton J. R., Insull W., Jr., Rosenfeld M. E., Schaffer S. A., Schwartz C. J., Wagner W. D., Wissler R. W., Circulation, 89, 2462-2478 (1994).

28) Stary H. C., Chandler A. B., Dinsmore R. E., Fuster V., Glagov S., Insull W., Jr., Rosenfeld M. E., Schwartz C. J., Wagner W. D., Wissler R. W., Circulation, 92, 1355-1374 (1995).

29) Shiomi M., Ito T., Hirouchi Y., Enomoto M., Ann. N.Y. Acad. Sci., 947, $419-423$ (2001).

30) Shiomi M., Ito T., Hirouchi Y., Enomoto M., Atherosclerosis, 157, $75-84$ (2001)

31) Newby A. C., Physiol. Rev., 85, 1-31 (2005).

32) Kuzuya M., Nakamura K., Sasaki T., Cheng X. W., Itohara S., Iguchi A., Arterioscler. Thromb. Vasc. Biol., 26, 1120-1125 (2006).

33) Ray B. K., Shakya A., Turk J. R., Apte S. S., Ray A., Circ. Res., 95, 1082-1090 (2004)

34) Burleigh M. E., Babaev V. R., Oates J. A., Harris R. C., Gautam S., Riendeau D., Marnett L. J., Morrow J. D., Fazio S., Linton M. F., Circulation, 105, 1816-1823 (2002).

35) Shiomi M., Ito T., Yamada S., Kawashima S., Fan J., J. Atheroscler Thromb., 11, 184-189 (2004).

36) Liang J., Liu E., Yu Y., Kitajima S., Koike T., Jin Y., Morimoto M., Hatakeyama K., Asada Y., Watanabe T., Sasaguri Y., Watanabe S., Fan J., Circulation, 113, 1993-2001 (2006). 